\title{
DIVERSITÉ DES LIENS ENTRE UN EMPRUNT ET SES ÉQUIVALENTS - LE CAS DE BINGE DRINKING
}

\author{
JEAN-FRANÇOIS SABLAYROLLES', ALENA PODHORNÁ- \\ POLICKÁ, ANDRZEJ NAPIERALSKI, JOHN HUMBLEY
}

\begin{abstract}
Diversity of relations between a loanword and its equivalents - the case of binge drinking

The objective of this article is to study the relations between a borrowing and its various native equivalents in the form of the explanatory text between them. The borrowing chosen for analysis is the term designating what is seen as a new threat to public health, i.e. binge drinking, which came to prominence in English-speaking countries. Typically, this is presented in the French, Polish and Czech corpus analyzed in various forms such as: binge drinking, which may be translated as [native equivalent]. It is shown that a whole range of presentations is possible, from the most specific to the most general and it is argued that the type of presentation is indicative of the degree of lexicalization of the native equivalent.
\end{abstract}

Keywords : Loanword, equivalent, explanation, typology

Mots-clés : Emprunt, équivalent, glose, typologie

\section{Introduction}

Les relations entretenues entre les équivalents d'un emprunt et l'emprunt lui-même sont nombreuses et variées, que l'équivalent soit néologique ou non. Dans cet article, nous proposons une typologie de ces relations attestées dans différents corpus telles qu'elles se manifestent dans les trois langues étudiées. L'emprunt retenu pour cette analyse est binge drinking, phénomène de société aux implications multiples, bien attesté en français, en polonais et en tchèque, première manifestation linguistique de toute une série de troubles d'un comportement addictif comme binge watching, binge eating ou binge shopping qui feront l'objet de travaux futurs. Les résultats observés pour binge drinking semblent proches de ceux relevés pour les autres emprunts étudiés dans le cadre du pré-

1 Jean-François Sablayrolles (25 mai 1951 -11 décembre 2020) n’a pas eu le temps de terminer cet article mais c'est lui qui a effectué toutes les recherches dans les archives d'Europresse, qui ont servi de point de départ pour les dépouillements en polonais et en tchèque. C’est lui qui a voulu axer cette étude sur les différentes configurations des segments glosateurs entre emprunt et proposition d'équivalent, prolongeant ainsi une problématique qu'il avait creusée avec Agnès Steuckardt. 
sent projet, en particulier de couch $^{-2}$, permettant une focalisation/orientation spécifique dans ce volume pour chaque étude de cas.

\section{Buts et méthodes}

La grille d'analyse proposée ici est issue d'un classement préalable des données obtenues pour le français, appliqué par la suite aux attestations recueillies pour le polonais et pour le tchèque. Le but de la présente étude étant de rendre compte de l'assimilation éventuelle de l'équivalent d'un emprunt, signalée par la formulation du segment glosateur, nous avons adopté une classification qui tient compte a posteriori des réflexions de Josette Rey-Debove (1998) et de Marie-Laure Pflanz (2014) qui, elles, s'intéressent à la lexicalisation de l'emprunt. Au lieu de se focaliser sur les anglicismes, nous cherchons à comprendre comment les explications qui introduisent l'équivalent fournissent un indice de lexicalisation de ce dernier. Dans la présente étude, nous poursuivons les recherches initiées par Agnès Steuckardt et ses collègues sur la glose (Steuckardt et Niklas-Salminen 2003, Steuckardt et Honoré 2006) surtout par rapport aux emprunts et « leurs équivalents autochtones " (Hildenbrand et al. 2016) et sur le langage des conduites « délictueuses, excessives ou à risque », par deux des présents auteurs (Sablayrolles et Napieralski 2016).

Nous partons de l'hypothèse que l'analyse des différents types de gloses (ou segments glosateurs selon Steuckardt et Niklas-Salminen $(2005: 6)$ ) comportant un équivalent est susceptible de fournir des indices quant au degré de lexicalisation de l'équivalent autochtone. La focalisation de notre analyse est donc orientée vers les moyens linguistiques, y compris typographiques, par lesquels les équivalents de l'emprunt sont reliés à l'emprunt lui-même. L'ordre de la présentation adoptée ici pour les trois langues tente d'aller des relations les plus explicites linguistiquement à celles qui le sont moins.

\subsection{Corpus}

Puisque le but de l'étude est d'établir une typologie des relations textuelles entre emprunt et équivalent, le corpus de départ choisi pour le français est constitué à partir des archives d'Europresse ${ }^{3}$, source qui permet de remonter relativement loin dans le passé, et de ce fait, de pouvoir se focaliser sur la réception de l'emprunt dès ses premières attestations, vers 2000. Il ne permet pas en revanche de tenir compte des fréquences, moins pertinentes dans le contexte de l'élaboration d'une typologie.

Le résultat de la requête binge-, formulée pour le français dans Europresse, est de 2635 occurrences le 21. 04. 2020 (requête limitée au français de France), mais l'examen des premières centaines d'occurrences montre l'existence d'un certain nombre de doublons, voire de triplons (un énoncé identique est présent plusieurs fois). Si l'on tient compte de ces répétitions et d'autres emprunts comportant binge- (binge watching en particulier), le nombre d'énoncés différents diminue considérablement, mais il reste néanmoins important.

2 Voir l'article « Diversité des composés en couch, de leurs équivalents et de leurs commentaires » dans ce volume.

3 http://www.europresse.com/fr/. 
L'absence de corpus strictement comparable pour les trois langues empêche toute comparaison basée sur les fréquences, mais puisque le but de l'étude est d'établir une grille d'analyse, cette carence n'est pas un inconvénient.

Le corpus polonais ${ }^{4}$ est constitué d'une recherche effectuée directement sur google.pl, sur la plateforme Sketch Engine (Polish Web 2012 (plTenTen12), lemmatisé par RFTagger) ${ }^{5}$ ainsi que dans les archives de presse disponibles en ligne, à savoir des journaux Gazeta Wyborcza - gazeta.pl, Rzeczpospolita - rp.pl, Wprost - wprost.pl, Fakt - fakt.pl.

Effectuer une recherche similaire dans un équivalent d'Europresse pour la langue tchèque, Anopress ${ }^{6}$, ne permettrait pas d'obtenir des résultats comparables parce que son moteur de recherche ne permet de visualiser que cinq années de suite (de plus, les nombreuses répétitions des mêmes textes ou encore des textes dans d'autres langues que le tchèque compliquent le bilan statistique). Le Corpus national tchèque propose cependant une alternative intéressante qui permet d'opter entre les corpus écrits et les corpus oraux,

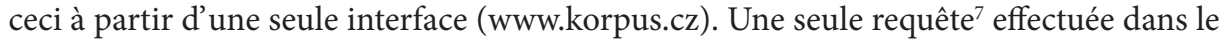
plus grand corpus écrit pour la langue tchèque, le syn $v 8$, concernant les composés en binge-, permet d'obtenir 109 résultats (sur 5 milliards de mots, 5G), témoignant de l'usage dans la presse tchèque depuis 1997. Ce petit échantillon a été complété par une recherche dans les différents corpus web disponibles sur la plateforme Sketch Engine (notamment 10G CzechTenTen et $16 \mathrm{M}$ (million de mots) Timestamped JSI web corpus 2020-09 Czech), sur le site des corpus de la famille Aranea (dont le plus grand corpus (taille plus de 7G) est Araneum Bohemicum Maximus) et dans online-now, un tout nouveau corpus-fleuve du web tchèque, lancé en novembre 2020 par le Corpus national tchèque (preque $1 \mathrm{M}$ de mots) pour arriver à plus de deux autres centaines d'autres occurrences, qui témoignent de la vivacité des adaptations du composé en binge- dans la langue tchèque.

Toujours est-il que dans les trois langues nous allons nous focaliser uniquement sur binge drinking, qui est très souvent suivi d'une glose, d'un équivalent ou d'une marque qui signale son statut d'emprunt, manifestations qui feront l'objet de l'étude qui suit.

\subsection{Note sur la chronologie}

Le binge drinking est un phénomène qui s'est manifesté tout d'abord dans les pays de langue anglaise en tant que problème de société et de santé publique. Les occurrences de l'emprunt binge drinking dans la presse française datent du début des années 2000, la première de 1998 dans un contexte américain, mais les mentions ne deviennent fréquentes qu'à partir de 2004. Sur le plan de la terminologie officielle, "FranceTerme » préconise beuverie express depuis 2013. Pour le polonais, les occurrences sont bien plus tardives (à partir de 2011) et aparaissent dans un contexte qui présente ce phénomène

Recherche et résultats datant du 30 septembre et $1^{\text {er }}$ octobre 2020.

Sketchengine.eu (https://app.sketchengine.eu/\#dashboard?corpname=preloaded\%2Fpltenten 12 _rft1). Ce moteur de recherche a été utile pour accéder aux sites Internet sur lesquels se trouvaient les occurrences de binge drinking. Dans le cas de la recherche pour la langue polonaise, nous considérons uniquement les attestations qui figurent sur les sites Internet.

6 https://monitoring-anopress-cz.ezproxy.muni.cz/Anopress.

7 En filtrant les noms propres grâce à une requête assez complexe, à savoir : [word=»[bB]inge.

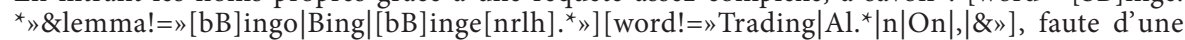
absence de lemmatisation (les composés en binge- ne font pas encore partie des dictionnaires intégrés). 
comme une menace venue de l'Ouest. Pour le tchèque, les attestations de binge drinking les plus anciennes datent de 2004 et apparaissent de manière ponctuelle dans la presse tchèque, sans preuve de circulation en dehors des explications journalistiques décrivant le phénomène sociétal.

\subsection{Difficultés pour trouver les équivalents}

La typologie qui suit a été élaborée à partir des exemples recueillis en français. Pour certaines catégories (celles qui se fondent sur la ponctuation, les guillemets, les parenthèses, par exemple), les formulations équivalentes en tchèque et en polonais ne posent pas de problème, mais lorsqu'il s'agit d'explication, le degré d'équivalence peut être problématique. Celui-ci repose tant sur le choix de la lexie pour le registre employé (quel équivalent synonymique choisir parmi toutes les formes disponibles) que sur le choix de la construction syntaxique adoptée pour la traduction (la traduction vise par-dessus tout à conserver le sens le plus précis d'un énoncé sans tenir compte à tout prix de l'ordre des mots dans la phrase ou de l'ordre des propositions). Lors de la traduction d'une phrase du polonais ou du tchèque vers le français, n'est-il pas un peu aléatoire de choisir parmi les verbes quasi-synonymiques 'appeler,' 'désigner' ou 'surnommer' pour un verbe polonais/ tchèque possédant le sens similaire ? Le problème qui apparaît pour le modus operandi de cette recherche est la question de l'équivalence de la traduction du discours à partir d'une langue étrangère. Une traduction peut-elle être considérée comme suffisamment équivalente au texte d'origine pour subir une analyse aussi détaillée ? En regardant les équivalents proposés pour le binge drinking (binge en anglais faisant référence à l'intensité et à la quantité) dans la terminologie officielle française, on remarque que c'est la rapidité qui est mise en évidence (le cas de l'adverbe express dans beuverie express). Dans le cas du polonais, c'est plutôt l'excès qui est invoqué (boire à tomber, boire à mort, boire en excès, etc.).

Cela dit, il faut être conscient que les données pour les langues tchèque et polonaise sont des adaptations qui peuvent faire débat en matière d'objectivité. Le cas du deuxième exemple polonais $(2 \mathrm{pl})$ montre que le participe présent du verbe 'désigner' dans la traduction en français est le résultat de la traduction et non pas de l'existence du verbe 'désigner' dans la phrase originale en polonais. Un problème similaire est évoqué dans l'exemple infra (11tch).

Dans de nombreux articles, dans les trois sous-corpus, la lexie binge drinking apparaît avec une glose signalisant directement la langue source (ang.). Toutefois, ce n'est pas le cas de tous les exemples, car on retrouve aussi l'utilisation des guillemets. L'exemple dans la glose ${ }^{8}$ est particulièrement édifiant du fait que le binge drinking est cité au même titre que five o'clock tea ou fish and chips comme une spécialité britannique : Ironie ? Évolution des mœurs? Le temps nous le montrera.

8 Pod względem etnicznym jest to jednak miejsce znacząco różniące się od typowej Anglii, którą kojarzymy z five o'clock, fish and chips czy binge drinking. [D'un point de vu ethnique, c'est un endroit qui diffère beaucoup de l'Angleterre typique, celle que nous connotons avec le five o'clock, fish and chips ou binge drinking] (tvp.info - https://www.tvp.info/46109885/wielka-brytania-synowie-imama -abu-hamzy-aresztowani/, consulté le 30. 09. 2020). 
Presque chaque article du corpus polonais fournit un équivalent ou une explication du phénomène. On retrouve ainsi des formes lexicales comme : maraton picia 'marathon de beuverie', chlanie 'biture', picie na umór 'boire à mort', upijanie się 'se saouler', picie do upadkego 'boire à tomber', pijaństwo 'saoulerie', picie na akord'cuite express', picie w nadmiarze 'boire en excès'. Deux formes dominent en ce qui concerne les équivalents dans la langue polonaise, picie na umór 'boire à mort' et la forme plus euphémique upijanie się 'se saouler', il faut signaler que l'équivalent proposé par Google picie do upadłego 'boire à tomber' n'apparaît qu'une seule fois. Dans le corpus tchèque, les équivalents autochtones les plus fréquents semblent être : nárazové pití 'consommation/beuverie d'un seul coup', mettant l'accent sur la rapidité et sur la limite temporelle de l'action (lit. nárazově = 'en choc, en rafales'), pití do nèmoty 'consommation/beuverie à la mort' (lit. 'jusqu'à en devenir sourd') qui officialise en quelque sorte une locution typiquement familière, mettant l'accent sur la conséquence de l'action ou encore problém nezřizeného pití 'problème d'une consommation/beuverie effrénée', où la synapsie anglaise reçoit un caractère terminologique grâce à l'antéposition du mot 'problème'.

L'interrogation d'un corpus brut nécessite la prise en compte du bruit. Même en précisant bien la langue de l'enquête (français, tchèque ou polonais), on obtient sur Google beaucoup de textes en anglais. De plus, plusieurs occurrences relèvent des noms propres, comme par exemple Hildegard von Binge Drinking, relevé lors d'une enquête en polonais, qui renvoie à une artiste allemande de musique électronique, jeu de mots sur l'austère Hildegard von Bingen.

\section{Analyse des données}

\subsection{Traduction avouée (et parfois commentée)}

La configuration 1 est la relation la plus explicite, celle qui fait mention d'une traduction de l'emprunt à l'aide précisément d'une forme du verbe traduire ou de son équivalent en polonais tlumaczyć et en tchèque přeložit (contrairement au tchèque, le substantif (traduction) n’a pas été trouvé dans le corpus français, mais il pourrait bien sûr être employé). C'est sans doute le participe passé passif traduit par qui est la forme la plus neutre, faisant abstraction de l'agent qui traduit, et utilisée à maintes reprises pour cet usage $(1 \mathrm{fr})^{10}$.

(1fr) Le binge drinking, appellation venue d'outre-Manche au début des années 2000, traduit par « biture express » ou " alcool défonce ${ }^{11}[\ldots]$

9 Nous hésitons entre " consommation » et « beuverie " dans la traduction parce que le premier mot est stylistiquement plus élevé que pití, terme assez neutre en tchèque (il s'agit d'une nominalisation du verbe «boire » pit).

10 Pour ne pas surcharger l'article d'informations non pertinentes pour son objectif, les références des exemples n'ont pas été reproduites. En revanche, pour faciliter la lecture, les marqueurs linguistiques en question pour chaque exemple ont été mis en gras.

11 Dans cet exemple, comme dans tous les autres, l'utilisation des guillemets est celle de la source. Parfois l'emprunt est mis entre guillemets, parfois non. Il en va de même pour l'équivalent ou les équivalents. Ce qui donne quatre situations possibles : les deux éléments entre guillemets, aucun des 
Il arrive néanmoins que des précisions sur la langue (2fr), voire des jugements sur la qualité de la traduction dans cette langue (3fr) et (4fr) accompagnent cette formulation.

(2fr) $[\ldots]$ " binge drinking ", traduit en français par " intoxication alcoolique aiguë », «biture expresse » ou « alcool défonce » $[\ldots]$

(3fr) C'est le « Binge drinking », traduit en bon français par « biture express ».

(4fr) $[\ldots]$ « binge drinking ». Traduit en français bien de chez nous, ça devient la « biture express $»,[\ldots]$

Il est également possible de retrouver des occurrences où la traduction est nuancée et présentée comme le choix d'un équivalent parmi plusieurs disponibles dont chacun comporte au moins un sème du sémème de la lexie anglaise binge drinking. Dans l'exemple (1tch), il est également intéressant de voir le glissement du statut de la locution en langue anglaise :

(1tch) Binge drinking, česky např. „nezř́zené pití, je výraz, který z odborné angličtiny pomalu mizí a nahrazuje ho neutrální „heavy episodic drinking“. [Binge drinking, en tchèque p. ex. "beuverie sans limites » est une expression qui est en train de disparaître de la terminologie anglaise pour être remplacé par un terme plus neutre « heavy episodic drinking $]^{12}$.

Une autre forme neutre recourt à l'emploi de la construction pronominale de sens passif, mais la seule occurrence de ce type que nous ayons relevée (5fr) est modifiée par un adverbe de manière à valeur négative, difficilement, sans que la raison de cette difficulté soit mentionnée et ce jugement paraît quelque peu gratuit, puisque l'équivalent introduit semble plutôt pertinent même si, apparu assez tôt, il n'a pas rencontré le succès auquel on aurait pu s'attendre ${ }^{13}$. Cette synthèse néologique, pour reprendre le terme employé par Loïc Depecker (2001), correspond en effet bien à la pratique nouvelle qui s'est largement répandue.

(5fr) $[. .$.$] le « binge drinking ». Cette expression désigne un véritable fléau et se traduit$ difficilement par « la course à la cuite».

Un jugement de valeur négatif est également exprimé dans (6fr) où le verbe traduire est employé à l'infinitif, précédé du verbe modal pouvoir au conditionnel.

(6fr) Le binge drinking - que l'on pourrait traduire en mauvais français par « biture express $»[\ldots]$

deux ou un seul des deux, soit l'emprunt, soit l'équivalent, sans qu' aucune logique n'apparaisse. Cette diversité dans l'emploi « [d] es marqueurs d'une certaine forme d'autonymie » (Pflanz 2014 : 165) que sont les guillemets révèle la différence de points de vue des locuteurs sur ces mots qu'ils emploient. En revanche, les italiques ne sont pas employés.

12 Vesmír, 2013 (mensuel).

13 Apparu en 2004, seules onze occurrences sont indiquées, la dernière remontant à 2012. 
Dans le corpus tchèque, le verbe «traduire» (přeložit) est relevé dans deux exemples, accompagné à chaque fois d' un adverbe qualitatif qui montre une volonté de traduire «le plus convenablement possible» (nejlépe) ou de traduire « librement » (volně) :

(2tch) Pokud jste se v posledních třech měsících $\mathrm{v}$ průměru jednou týdně přejedli, tak už se na vás vztahuje porucha „binge eating“. Což lze asi nejlépe přeložit $v$ souvislosti se známějším „binge drinking“ (kdy to do sebe flamendři lijí jako do sudu) - takže tř̌eba jako „návalové obžerství “. [Si vous avez trop mangé une fois par semaine en moyenne durant les trois derniers mois, vous avez affaire à la dysfonction « binge eating ». La solution la plus convenable de traduire ceci serait en relation avec le teme plus connu « binge drinking » (quand les habitués des pubs se versent un coup après l'autre comme si leur ventre était un tonneau) - alors par exemple comme « afflux de gloutonnerie $»^{14}$.

(3tch) « „Binge drinking“, volně přeloženo jako pití v tazích... “ [ « Binge drinking », librement traduit comme beuverie en tournée... $]^{15}$.

À la différence du français, le corpus tchèque contient un substantif «traduction », précédé par le qualificatif «bonne » (správný prèklad):

(4tch) Českou metropoli oglosovala paní komisařka takto: „Praha je krásné město, které kazí jen hordy mladých mužů jezdících sem jen za pivem (bingedrinking! - správný překlad je zřejmě ,chlastat' nebo ,ožírat se' - pozn. autora) a prostitutkami." [La métropole tchèque a été glosée par madame la commissaire ainsi: „Prague est une belle ville, gâchée seulement par des hordes de jeunes hommes qui viennent ici juste pour la bière (bingedrinking! la traduction correcte est apparemment ,se saoûler' ou, ,se bourrer' - note de l'auteur) et pour les prostituées." $]^{16}$.

Ce qui semble surprenant dans le cas du corpus polonais, c'est le fait que le verbe « traduire » (przetłumaczyć, tłumaczyć) ne soit pas employé en relation avec binge drinking. On observe l'abréviation (tłum) dans certains cas, mais il s'agit plutôt du substantif tłumaczenie et non du verbe.

D'autres emplois de l'infinitif, actif (7fr) ou pronominal de sens passif comme supra (8fr), précédé du modal pouvoir sont attestés sans commentaire, autre que l'indication de la possibilité. Dans le corpus polonais, on retrouve l'emploi de l'infinitif actif (1pl).

(7fr) [...] le binge-drinking que l'on peut traduire par biture express ou alcool-défonce. ${ }^{17}$

(8fr) Le binge drinking peut se traduire par beuverie express.

(1pl) To legalizacja marihuany ma również wpływ na tzw. binge drinking, co na polski można przetłumaczyć jako maratony picia albo - mówiąc wprost - chlanie. [C'est la dépé-

\footnotetext{
Hospodárské noviny, 2013 (quotidien).

Mladá fronta DNES, 2013 (quotidien).

Lidové noviny, 2005 (quotidien).

Le pronom indéfini on a la même fonction de gommer l'identité de l'agent que les tournures passives ou pronominales de sens passif.
} 
nalisation de la marijuana qui affecte aussi ce qu'on appelle le binge drinking, ce qu'on peut traduire en polonais comme marathon de beuverie ou tout simplement biture. ${ }^{18}$

L’infinitif est aussi employé dans sa valeur injonctive (9fr) et (10fr) :

(9fr) [...] pratique répétée dite du «binge drinking » (traduire par «beuverie express »). (10fr) [...] lutter contre le « binge drinking », à traduire par « cuite express ».

La valeur injonctive s'exprime aussi naturellement par des impératifs, à la première personne du pluriel (11fr) ou à la deuxième (12fr).

(11fr) On appelle ça le binge drinking. Traduisons la biture express.

(12fr) Binge drinking... traduisez par beuverie express ou biture express.

Le mode indicatif est peu employé, mais on le trouve en particulier pour imputer la traduction à un groupe de locuteurs (13fr), autorisés en l'occurrence (spécialistes, etc.), ou à une personne précise, reprise en anaphore par le pronom il (14fr). Parfois cette personne précise peut être le locuteur même qui propose sa traduction, en employant non pas le verbe traduire mais le verbe proposer (15fr).

(13fr) [...] le " binge drinking ", que les spécialistes traduisent par «biture express ».

(14fr) [...] lutte contre le «binge drinking » (qu'il traduit par «biture express »).

(15fr) Je trébuche sur un «binge drinking ». Un anglicisme qui, pour l'heure, n'a reçu qu' une proposition - une "biture fissa " - pour désigner ce phénomène qui consiste à ingérer de l'alcool en un temps record. Je songe à " hyperalcoolisation » mais la notion de rapidité n'est pas restituée. Je propose une "beuverie express.$^{19}$

Le corpus tchèque comporte également l'exemple intéressant d'une tentative de définition, et non de traduction, prenant alors le binge drinking pour notion/concept à définir et non à traduire (sans succès dans l'article).

(5tch) Přesná definice tzv. Binge Drinking neexistuje. [Une définition précise d'un soi-disant Binge Drinking n'existe pas.] $]^{20}$.

\subsection{Une dénomination équivalente}

La configuration 2 est celle d'une dénomination équivalente. De même que le recours au verbe traduire implique un point de vue métalinguistique ou simplement épilinguistique, celui à des termes de dénomination révèle un recul conscient du locuteur et des

18 https://spidersweb.pl/2020/01/legalizacja-marihuany-w-stanach-zjednoczonych-wplyw-na-studentow.html/, consulté le 30. 09. 2020.

19 Biture fissa n'est attesté que dans ce seul exemple, daté de 2011, dans la base Europresse, alors que beaucoup d'équivalents circulaient depuis longtemps. Une requête sur le moteur de recherche Google donne 738 résultats, ce qui n'est pas énorme.

20 Hospodářské noviny, 2008 (quotidien). 
emplois autonymiques. Là encore le participe passé passif du verbe, appeler, zwany, tak $z$ wany (plus utilisé comme acronyme tzw.) en polonais, zvaný, tak zvaný ou, plus fréquemment, takzvaný en tchèque (raccourci en $t z v$. de manière identique qu'en polonais), se taille la part du lion dans ce type d'emplois, dont nous ne donnons que quelques exemples parmi beaucoup d'autres. Ils sont souvent accompagnés d'adverbes comme aussi, encore, etc. (16fr) et (17fr), więc en polonais ( $2 \mathrm{pl}$; absent dans le corpus tchèque) ou d'un adverbe comportant un jugement (18fr).

(16fr) Aussi appelé « biture express » ou «beuverie express », le binge drinking consiste à boire un maximum d'alcool en un minimum de temps.

(17fr) L’incitation au «binge drinking» (encore appelé « biture express ») [...]

(18fr) [...] phénomène anglo-saxon dénommé « binge drinking », plus communément appelé chez nous « cuite express ».

(2pl) Unikamy natomiast, popularnego tak w Polsce, tzw. „binge drinking” a więc picia w nadmiarze, w celu upicia się. [Nous évitons cependant le soi-disant "binge drinking ", très populaire en Pologne, alors processus désignant l'action de boire en excès dans le but de se saouler. $]^{21}$

La dénomination s'exprime aussi avec le verbe surnommer (encore au participe passé passif (19fr), ou encore avec le verbe désigner (au participe présent (20fr) ou à l'indicatif dans une relative $(21 \mathrm{fr}))^{22}$.

(19fr) L'alcoolisation massive, connue sous le nom anglais de « binge-drinking » ou beuverie massive, encore surnommée biture express, se répand [...]

(20fr) [...] pratiques de « binge drinking » anglo-saxon désignant une alcoolisation massive et rapide $[\ldots]$

(21fr) La « beuverie express » est désormais l'expression française qui désigne le «binge drinking " anglo-saxon, selon un avis $[\ldots]$

Une des possibilités de traduire la phrase du corpus polonais est de s'aider du participe du verbe désigner à la voix passive $(3 \mathrm{pl})$.

(3pl) W tej grupie wiekowej, kiedy wielu młodych ludzi studiuje, dominuje wzorzec picia określany z języka angielskiego jako binge drinking. Polega on na intensywnym upijaniu się, czyli wypijaniu bardzo dużych ilości alkoholu w krótkim czasie. [Dans ce groupe d'âge, quand il y a beaucoup de jeunes qui étudient, le fait de consommer l'alcool est désigné par l'anglais binge drinking. Il consiste à se saouler d'une manière intense, c'est-à-dire en buvant d'énormes quantités d'alcool en un temps limité.] ${ }^{23}$

21 https://www.ofeminin.pl/fitness-i-zdrowie/dolegliwoscichoroby/jak-zyc-zdrowo-i-uchronic-sieprzed-rakiem-radzi-nasza-ekspertka-dietetyczka-i/14cvc9l, consulté le 01. 10. 2020.

22 Si les linguistes distinguent ordinairement la dénomination (avec l'utilisation du nom porté par ce dont on parle) et la désignation, à l'aide d'une périphrase ou d'un simple geste, le commun des mortels a tendance à assimiler les deux processus et désigner est alors souvent synonyme de dénommer. C'est nettement le cas dans les deux exemples (20fr et $21 \mathrm{fr}$ ).

${ }^{23}$ https://tvnmeteo.tvn24.pl/informacje-pogoda/ciekawostki,49/mlode-polki-pija-jak-mezczyzni, 99709,1,0.htm, consulté le 01. 10. 2020. 
Ce sont enfin des noms (terme (22fr), équivalent (23fr) ou version française (24fr)) qui sont utilisés pour introduire les équivalents français.

(22fr) Le « Binge Drinking », terme anglo-saxon qui peut se traduire par « conduite d'alcoolisation massive dans un temps très court ", connu également sous le terme "d'intoxication alcoolique aiguë ».

(23fr) [Binge drinking] a désormais son équivalent en bon français, « beuverie express ", selon un avis paru dimanche au Journal officiel.

(24fr) [...] thème de la <sic> binge drinking, biture express dans sa version française.

Dans les corpus polonais et tchèque, cette catégorie est largement représentée par les adjectifs zwany ( « appelé ») / zvaný ( « appelé ; dit») ou, plus souvent, tak zwany ( "soi-disant ») / takzvaný (« soi-disant»). Comme nous l'avons vu, ce dernier est le plus souvent raccourci en $t z w / t z v$.

(4pl) „[...] w jakich krajach największy odsetek 15- i 16-latków praktykuje tzw. „binge drinking“, czyli upijanie się na umór [...]. [[...] dans quels pays il y a le plus grand pourcentage de 15-16 ans qui s'adonnent au soi-disant „binge-drinking”, donc le fait de boire à mort $[\ldots].]^{24}$

(5pl) „Internetowa rozrywka doskonale się wpisała w lokalną rzeczywistość, gdzie upijanie się na umór (tzw. binge drinking) jest traktowane jak sport narodowy." [Les loisirs venus d'Internet

s'est parfaitement inscrit dans la réalité locale où l'action de boire à mort (soi-disant. binge drinking) est considérée comme un sport national. ${ }^{25}$

(6pl) „Stwarza to zupełnie nowe horyzonty dla ludzi uprawiających tak zwany binge drin-

king..." [Cela crée des horizons nouveaux pour les personnes qui s'adonnent au soi-disant binge-drinking.... $]^{26}$

(6tch) „Přitom by se měli spíš zaměřit na pivovary, které prodávají ve výprodeji 24 piv za sedm liber - tam leží problém zvaný binge drinking, " upozorňuje Martin Dickie na lehce nekalou konkurenci, která vybízí k bezbřehému kalení. [ "Ils devraient se concentrer davantage sur les brasseries qui vendent en soldes 24 bières pour sept livres - c'est là que repose le problème appelé le binge drinking ", dit Martin Dickie, soulignant la concurrence un peu déloyale qui encourage la beuverie sans limites. $]^{27}$

(7tch) Ze zdravotního hlediska je pro mládež nejnebezpečnější nárazové pití většího množství alkoholu, takzvané binge-drinking. [Du point de vue de la santé, le plus dangereux pour les jeunes est la consommation d'un seul coup d'une plus grande quantité d'alcool, le soi-disant binge-drinking. $]^{28}$.

(8tch) Politici zákonem reagovali na rychlé zvyšování počtu př́padů tzv. binge drinking, kdy se mládež snaží co nejrychleji opít. [Par l'adoption d'une loi, les politiciens ont réagi

\footnotetext{
24 https://facet.wp.pl/tam-zyja-najbardziej-zdemoralizowane-kobiety-w-europie-6006482324271745g/2, consulté le 28. 12. 2020.

25 https://www.wprost.pl/tygodnik/436386/null, consulté le 28. 12. 2020.

26 https://wiadomosci.wp.pl/jak-zdejmiesz-majtki-dostaniesz-darmowego-drinka-6036808117609601a, consulté le 28. 12. 2020.

Strategie, 2010 (mensuel).

Instinkt, 2015 (hebdomadaire).
} 
à la hausse des cas d'un soi-disant binge drinking quand la jeunesse s'efforce à se rendre ivre le plus vite possible. ${ }^{29}$

\section{3 Équivalence fondée sur la signification}

La configuration 3 est fondée sur la signification. Le locuteur recourt aussi à des verbes indiquant plus ou moins explicitement un rapport fondé sur une signification identique de l'emprunt et de l'équivalent ou des équivalents. Les plus explicites d'entre eux sont précisément les verbes signifier (25fr) et vouloir dire (26fr) pour le français. On retrouve le verbe oznaczało (= "signifier") à l'imparfait dans une traduction d'une occurrence du corpus polonais $(7 \mathrm{pl})$, où il est question de binge watch ${ }^{30}$ qui est expliqué par rapport à binge drinking. Dans le corpus tchèque, le verbe parallèle à l'exemple polonais, označit est relevé dans la description d'une dérivation, de binge drinker (mais avec le sens plutôt de « qualifier de, marquer comme »).

(25fr) [...] phénomène venu d'Outre-Manche appelé binge drinking : l'expression signifie «boire pour se rendre minable » ou « course à la cuite ». ${ }^{31}$

(26fr) [...] lutte contre le « binge drinking ». Littéralement, ça veut dire «biture express », mais ce n'est pas très joli ; il faut qu'on lui trouve un autre nom.

(7pl) Binge-watch - moim skromnym zdaniem, to uroczy twór. Do niedawna istniało sobie tylko «binge-drinking», stowo pramatka, które oznaczało "picie na umór. [Binge-watch - c'est à mon avis une création lexicale charmante. Jusqu'à récemment il n'existait que le « binge drinking », mot ancestral, qui signifiait «boire à mort ». $]^{32}$

(9tch) Ne každého, kdo si v pátek popovídá u pár dvanáctek, lze označit jako binge drinkera... [On ne peut pas qualifier de binge drinker tous ceux qui bavardent les vendredi autour des bières de 12 degrés.... $]^{33}$.

Les verbes comprendre (27fr) et entendre (au sens de « comprendre ») (28fr), sont utilisés plusieurs fois, à l'impératif de la deuxième personne. Les corpus polonais et tchèque ne contiennent aucune reformulation de ce type.

(27fr) [...] on s'alcoolise massivement. Du binge drinking, comprenez : beuverie express. (28fr) [...] partir en guerre contre le « binge drinking ", entendez la cuite express.

Un appel de type conniventiel à l'interlocuteur / lecteur, sous la forme de la deuxième personne est également présent avec une forme du verbe savoir, à l'indicatif présent (29fr).

29 Právo, 2009 (quotidien).

30 Binge watch vient de binge watching c'est-à-dire d'une pratique qui consiste à regarder des épisodes d'une série (ou de films d'un univers commun) l'un après l'autre pendant une période de temps continue, le plus souvent toute la nuit.

31 Notons que le premier élément donné après le verbe signifier est bien éloigné de la source et contrevient aux règles (la tête est un verbe à l'infinitif et non un nom) à la différence du second, bien meilleur à tous égards.

32 https://natemat.pl/blogi/aleksandralojek/113491,o-piersi-bocznej-czyli-nowe-slowka-w-jezyku-angielskim\#, consulté le 01. 10. 2020.

33 Psychologie dnes, 2014 (mensuel). 
(29fr) On connaissait le «binge drinking» - vous savez, cette mode répandue chez les ados, la beuverie express.

Enfin, le verbe considérer comme, au participe passé passif, peut être rangé dans cette section dans la mesure où il s'agit bien d'une opération intellectuelle fondée sur le sens (30fr). Ici aussi, pas d'attestation dans le corpus tchèque.

(30fr) Et le phénomène du «binge drinking » considéré comme une «biture express » $[\ldots]$.

\section{4 Équivalents introduits par ou, autrement dit, c'est-à-dire, etc.}

Plusieurs outils linguistiques sont utilisés pour mettre en relation deux termes équivalents, en particulier un emprunt et un ou plusieurs de ses équivalents dans une autre langue. Le plus fréquent et le plus simple est sans doute la conjonction de coordination ou (31fr) et (32fr). Dans le corpus polonais, cela n’a pas été trouvé, alors que, dans la langue tchèque, ceci correspond à la conjonction de coordination au sens indentificatoire neboli (10tch).

(31fr) [...] le binge drinking, ou « course à la cuite».

(32fr) [...] phénomène du « binge drinking » ou biture express, ou encore « alcool défonce à gogo $"$.

(10tch) Je fakt, že na ostrovech se pije (ne-li chlastá) zničujícím způsobem. Tahle země dala světu výraz binge-drinking neboli pití s cílem co nejrychleji se ožrat do němoty. [Il est vrai qu'on boit (pour ne pas dire qu'on picole) sur les îles d'une manière destructive. Ce pays a donné au monde l'expression binge-drinking ou bien la consommation avec le but de se saoûler à mort. (lit. 'jusqu'à en devenir sourd'). $]^{34}$

Parmi les autres outils relevés une ou plusieurs fois, ont été employés autrement dit (33fr), c'est-à-dire (34fr) que nous retrouvons aussi dans le corpus polonais (les formes czyli (8pl) et takie jak (9pl) ont servi à la base pour la traduction), c'est (35fr), soit (36fr),

(33fr) On appelle ça le « binge drinking ». Autrement dit biture express.

(34fr) $[\ldots]$ « binge drinking » (c'est-à-dire une alcoolisation massive et rapide).

(35fr) Binge drinking, ça fait branché. C'est la cuite expresse <sic >, l'alcool défonce.

(36fr) La fête, avec ses excès d'alcool - le binge drinking, soit une beuverie express, fait des ravages.

(8pl) Nad Sekwaną wino spożywane jest w niewielkich ilościach do posiłków, podczas gdy na Wyspach popularny jest tzw. binge drinking, czyli picie na umór. [Au bord de la Seine on consomme le vin en petites quantités, juste pour accompagner le repas, cependant sur

34 Týdeník Květy (č. 35) (hebdomaire). 
les Îles (Angleterre - red) ce qui est populaire c'est ce qu'on appelle le binge drinking, c'est-à-dire boire à mort. ${ }^{35}$

(9pl) Ponieważ alkoholizm jest w języku potocznym synonimem uzależnienia, pewne zjawiska, rozpowszechnione przede wszystkim wśród młodzieży, takie jak binge drinking, czyli okazjonalne, szybkie upijanie się, nie były tradycyjnie zaliczane do zjawisk patologicznych. [Du fait que l'alcoolisme est considéré dans la langue familière comme synonyme de dépendance, il y a certains phénomènes, répandus surtout chez les jeunes, comme le binge drinking c'est-à-dire la cuite rapide, qui n'étaient pas considérés traditionnellement comme des phénomènes pathologiques. $]^{36}$

On a trouvé un emploi de l'adverbe donc (37fr), qui ne semble pas complètement naturel (il ne correspond guère aux emplois répertoriés de cet adverbe), mais dont la fonction de mise en relation d'équivalence entre deux termes en contexte est indéniable. L'introduction de l'équivalent à l'aide de l'adverbe donc a aussi été répertoriée dans le corpus polonais (10pl).

(37fr) [...] le rituel du binge drinking, donc de la «beuverie express ».

(10pl) We wspomnianych badaniach postanowiono sprawdzić ogólne samopoczucie psychiczne mieszkańców terenów wiejskich i częstość występowania u nich takich problemów jak depresja oraz znaczne nadużywanie alkoholu (w oryginalnej pracy użyto określenia binge drinking, a więc upijanie się, picie do upadłego). [Dans les études mentionnées il a été décidé de vérifier la condition psychique générale des habitants des territoires ruraux et la fréquence de l'apparition chez eux de problèmes tels que la dépression ou la surconsommation d'alcool (dans l'étude originale on a utilisé le terme binge drinking donc : se saouler ou boire à tomber. $]^{37}$

(11tch) Pokud jde o binge drinking, tedy pití do němoty,... [En ce qui concerne le binge drinking, c'est-à-dire/donc/alors/soit une beuverie à mort... $]^{38}$.

L'équivalent pragmatique de cette catégorie, la conjonction de coordination consécutive tedy est fréquente dans le corpus tchèque, nous nous résignons cependant à une traduction univoque. Il est intéressant de noter une combinaison fréquente de la construction tzv. emprunt, tedy:

(12tch) Celá debata se vede o tzv. binge drinking, tedy konzumaci alkoholu za účelem uvedení se co nejrychleji do značně povzneseného stavu. [Tout le débat porte sur le soi-disant binge drinking, c'est-à-dire la consommation d'alcool ayant pour but de se mettre dans un état assez avancé le plus rapidement possible $]^{39}$.

35 https://kobieta.onet.pl/zdrowie/choroby-wywolane-naduzywaniem-alkoholu-depresja-podagra -nowotwory-demencja/nb0fyye\#slajd-2, consulté le 01. 10. 2020.

36 https://zdrowie.gazeta.pl/Zdrowie/1,111848,8977972,Alkohol_i_alkoholizm.html, consultéle 01.10. 2020.

37 https://www.cenyrolnicze.pl/wiadomosci/wiesci-rolnicze/pozostale-wiesci-rolnicze/18221-rolnicy-narazeni-na-depresje-i-alkoholizm-badania-kanadyjskie, consulté le 30. 09. 2020.

38 Hospodárské noviny, 2015 (quotidien).

39 Mladá fronta DNES, 2007 (quotidien). 
Il arrive parfois que ce ne soit pas l'auteur de l'énoncé qui introduise l'équivalent mais un autre type d'énonciateur, ainsi qu'en atteste la mention " note de la rédaction » sous la forme siglée traditionnelle NDLR (38fr).

(38fr) [...] les pratiques comme le binge drinking (NDLR - beuverie express).

Le corpus tchèque comporte également ce type de traduction signée par l'auteur, avec le traditionnel pozn. autora (voir exemple (4tch) supra).

\subsection{Construction détachée, équivalent entre parenthèses}

Plusieurs types de constructions détachées sont utilisés dans la mise en relation de l'emprunt et de son ou ses équivalents. Nous distinguerons le recours aux parenthèses et à la simple apposition, entre virgules. Comme attendu, les cas les plus fréquents placent l'équivalent après l'emprunt - et nous commencerons par ces cas dans les sections 5 . et 6. - mais il arrive aussi, plus rarement et plus bizarrement, que ce soit l'emprunt qui se trouve dans la construction détachée, après le ou les termes autochtones. Des exemples en seront fournis dans les sections 7. et 8., avec également un cas en 10.

Innombrables sont les cas où un ou plusieurs équivalents sont placés entre parenthèses derrière le mot étranger, que celui-ci soit un xénisme - comme ce fut le cas pour binge drinking tant que l'utilisation de ce terme était réservée à une pratique proprement anglo-saxonne, inconnue ailleurs, avant son extension à de nombreuses autres régions du globe - ou qu'il soit un emprunt - comme binge drinking l'est devenu dans les langues des pays qui ont introduit cette pratique sociale d'alcoolisation massive et rapide pratiquée surtout par des jeunes en fin de semaine, avec des alcools forts. Nous n'en fournirons que quelques exemples introduisant divers équivalents, sans être exhaustif (39fr) à (43fr).

(39fr) [...] la culture du binge drinking ( « la course à la cuite ») [...]

(40fr) [...] le binge drinking (cuite express) [...]

(41fr) Marisol Touraine a récemment évoqué le «binge drinking » (alcoolisation massive et rapide).

(42fr) [...] moyen de lutter contre le « binge drinking " (l'alcool défonce).

(43fr) [...] phénomène du «binge drinking » (beuverie express).

Dans la langue polonaise, aucun exemple d'équivalent entre parenthèses pour expliquer le phénomène n'a été repéré. Pour le tchèque, deux occurrences de ce type ont été trouvées dans le corpus mais chaque fois, la parenthèse ne contient pas vraiment un équivalent tchèque proprement dit. Dans un premier exemple, la parenthèse comporte l'équivalent en allemand. Ceci remplit alors formellement la contrainte de cette catégorie 5 mais au niveau du contenu, cet exemple rentre également dans la catégorie 11 ci-dessous :

(12tch) „Západoevropský a americký koncept binge drinking (německy Rauschtrinken) popisuje fenomén jednorázové konzumace alkoholu s cílem opít se.“ [Le concept d’Europe 
de l'Ouest et d'Amérique du binge drinking (en allemand Rauschtrinken) décrit le phénomène de la consommation ponctuelle d'alcool avec le but de s'enivrer. $]^{40}$.

Dans le second exemple, la parenthèse contient une explication plutôt qu'un équivalent en voie de lexicalisation (voir l'exemple 2tch): „binge drinking“ (kdy to do sebe flamendřri lijí jako do sudu) [(quand les habitués des pubs versent un coup après coup comme dans un tonneau)].

Il est également à noter que la parenthèse peut contenir à la place d'un équivalent la prononciation de l'expression, comme le montre l'exemple suivant, tiré du corpus tchèque :

(13tch) Pak je tu ještě další fenomén, který ale žebříčky spotřeby nepopisují: nárazové pití, ve světě známé jako „binge drinking“ (čti ” bindž drinking “). [Puis il y a un autre phénomène que les tableaux de consommation ne décrivent pas : la consommation « cul sec", connue dans le monde comme «binge drinking " (lis/prononce «bind ̧̧ drinking ») $]^{41}$.

\subsection{Construction détachée, équivalent en apposition}

La mise en apposition, réalisée graphiquement par l'utilisation de virgules marquant le détachement, est également un procédé très fréquemment mis en action pour introduire un ou des équivalents à un terme étranger, avec déterminant, défini (44fr) et (45fr), ou indéfini (46fr) ou sans déterminant (47fr).

La construction par apposition existe aussi dans les cas des exemples tirés du corpus polonais $(11 \mathrm{pl})$ et du corpus tchèque (14tch). Pour l'exemple tchèque, il est intéressant de noter qu'il s'agit de la plus ancienne attestation de binge(-)drinking dans la presse tchèque (la lexie ne se décline pas encore). Le tchèque ne comporte pas d'articles, la question du caractère des déterminants est alors sans objet.

(44fr) Sus au binge drinking, la course à la cuite !

(45fr) Nous sommes également très préoccupés par le « binge drinking », l’alcool-défonce, [...]

(46fr) C’est le « binge drinking », une alcoolisation massive et rapide.

(47fr) [...] « Binge drinking », intoxication alcoolique aiguë, [...]

(14tch) Samotný britský premiér Tony Blair varoval národ před binge-drinking, rostoucím užíváním alkoholu, který označil za "novou britskou nemoc". [Le Premier ministre britannique Tony Blair lui-même a mis en garde la nation contre le binge-drinking, la consommation croissante de l'alcool, qu'il a qualifiée d'“une nouvelle maladie britannique“].42

Pour l'exemple polonais, quoique l'apposition ne soit pas souvent utilisée dans la langue courante, nous avons à faire ici au cas où il nous semble que la traduction appropriée pourrait se passer de conjonction.

Psychologie dnes, 2014 (mensuel).

Lidové noviny, 2016 (quotidien).

42 Attestation la plus ancienne de binge(-)drinking en tchèque dans le Corpus national tchèque, syn v8 (article du quotidien Hospodářské noviny du 22 novembre 2004). 
(11pl) Karierę robią binge drinking, czyli picie do nieprzytomności, oraz flatrate drinking - picie do oporu za kilkanaście euro. [Font fureur le binge drinking, boire jusqu'à l'inconscience ainsi que le flatrate drinking - boire jusqu'à l'excès pour quelques euros.] ${ }^{43}$

\subsection{Construction détachée, emprunt entre parenthèses après l'équivalent}

Les mêmes outils graphiques que sont les parenthèses et les virgules sont aussi employés dans les cas où le mot étranger figure après le mot autochtone. Cet ordre a priori illogique ne se trouve qu'à partir du moment où les équivalents ont suffisamment circulé dans la langue pour être employés d'abord, la mention du terme étranger ne venant ensuite que comme rappel de l'origine de leur émergence et de l'influence étrangère qui les a fait naître. Les exemples avec le terme étranger entre parenthèses après l'équivalent sont nettement moins nombreux que l'inverse vu précédemment, mais c'est néanmoins loin d'être anecdotique. Sur cette configuration et ce qu'elle signifie de l'implantation de l'emprunt, les réflexions de Pflanz (2014) sont fort pertinentes. Nous n'en donnons que quelques exemples avec des équivalents variés, de (48fr) à (51fr). Dans ce dernier cas, la langue d'origine est explicitement mentionnée.

(48fr) [...] la cuite express (binge drinking).

(49fr) Ce phénomène récent se rapproche de la « défonce alcoolique » hebdomadaire («binge drinking»).

(50fr) Cela pourrait expliquer le succès des sessions de beuverie express (binge drinking) chez les jeunes.

(51fr) [...] conduites à risque comme la « biture express » (« binge drinking » en anglais).

Notons que l'équivalent ainsi mis en valeur en français est soit le terme recommandé officiellement (biture express), soit une forme proche (beuverie express, cuite express...), soit des candidats à la lexicalisation.

La mise entre parenthèses de l'emprunt après l'équivalent semble une pratique habituelle dans les textes polonais, cela peut être illustré par trois exemples (12pl), (13pl) et (14pl).

(12pl) Choć zmiany w obrębie histonów zachodzą w sposób naturalny, naukowcy odkryli, że picie na umór (ang. binge drinking) powoduje nienaturalne modyfikacje, które negatywnie wpływają na funkcjonowanie DNA. [Quoique les changements dans les limites des histones se passent d'une façon naturelle, les scientifiques ont découvert que la cuite à mort (ang. binge drinking) provoque des modifications anormales qui influencent d'une façon néfaste le fonctionnement de l'ADN.] ${ }^{44}$

43 https://www.rp.pl/artykul/164822-Alkohol-zamiast-narkotyku.html, consulté le 15. 11. 2020.

44 https://tech.money.pl/medycyna/artykul/picie-na-umor-zmienia-aktywnosc-genow,45,0,1635885 .html, consulté le 30. 09. 2020. 
(13pl) Alkohol pomaga na ból tylko w stałych dawkach prowadzących do pijaństwa (ang. binge drinking - red.). [L'alcool aide à surpasser la douleur uniquement dans des doses stables qui mènent à la saoulerie (ang. binge drinking - NDLR). $]^{45}$

(14pl) Wypijanie dużych ilości alkoholu w krótkim czasie, tzw. picie na akord (z j. angielskiego binge drinking) jest popularne wśród studentów i ogólnie młodych ludzi w USA i krajach Europy. [La consommation d'une grande quantité d'alcool en un temps limité, ce qu'on appelle cuite express (de la langue anglaise binge drinking) est populaire parmi les étudiants et en général parmi les jeunes gens aux États-Unis et dans les pays européens. $]^{46}$

Dans le corpus tchèque, il est plus fréquent de trouver l'emprunt entre parenthèse après l'équivalent, à la différence du corpus français, que ce soit sans guillemets partout (15tch) ou avec des guillemets sur l'emprunt (16tch) ou sur l'équivalent (17tch).

(15tch) Americká literatura považuje za pití v tazích (binge drinking) dávku odpovídající asi 58 gramům $100 \%$ alkoholu [...]. [La littérature américaine considère comme beuverie en tournées (binge drinking) une dose équivalent à environ 58 grammes de l'alcool $100 \%$ $[\ldots]^{47}$

(16tch) Výrobci nemohou za to, že se např́klad pití do němoty („,binge drinking“) stává v některých evropských zemích jakýmsi módním trendem [...]. [Ce n’est pas la faute des fabricants si, par exemple, la consommation excessive d'alcool («binge drinking ») devient une sorte de mode dans certains pays européens [...] $]^{48}$.

(17tch) [...] důsledek módy tzv. „rychlého opití“ (binge drinking, viz [...]) [...conséquence de la mode d'une soi-disant « biture express » (binge drinking, voir...) $]^{49}$.

\subsection{Construction détachée, emprunt en apposition après l'équivalent}

Dans cette configuration, le terme anglais peut être seul mis en apposition (52fr) et (53fr) ou se trouver dans un groupe où il est introduit par le participe passé appelé (54fr).

(52fr) [...] contrecarrer le phénomène d'alcoolisation massive et rapide, le binge-drinking, $[\ldots]$

(53fr) La recherche de la défonce alcoolique, le « binge drinking », [...]

(54fr) $[\ldots]$ phénomène de « biture express », encore appelée « binge drinking », [...]

Pour le polonais comme pour le tchèque, cette catégorie est sous-représentée. Les quelques exemples sont toujours précédés par $t z w$. / tzv. (voir catégorie 2), ce qui semble être le signe de sa faible circulation dans la langue.

\footnotetext{
45 https://www.focus.pl/artykul/piwo-lagodzi-bol-lepiej-niz-paracetamol-jednak-nie-ma-rozy-bez -kolcow, consulté le 30. 09. 2020.

46 https://naukawpolsce.pap.pl/aktualnosci/news\%2C459687\%2Cupijanie-sie-w-mlodym-wieku -przyczyna-zmian-w-mozgu.html, consulté le 30. 09. 2020.

Lidové noviny, 2015 (quotidien).

Hospodářské noviny, 2008 (quotidien).

49 Vesmír, 2014 (mensuel).
} 


\section{9 Équivalence où l'emprunt est donné comme équivalent de l'équivalent !}

Paradoxalement, des énoncés abordant la dénomination du phénomène en recourant à des mots comme appelé (55fr) ou baptisé (56fr) semblent laisser croire que le mot étranger, binge drinking en l'occurrence, est second par rapport aux mots français qui seraient premiers. C'est aussi l'impression donnée par (57fr) et (58fr), mais dans une moindre mesure. Sans doute s'agit-il de formulations malheureuses, ne correspondant pas à ce que les scripteurs voulaient dire. En tout état de cause, les formulations de ce type sont assez rares.

(55fr) [...] la « cuite express », appelée aussi « binge drinking ».

(56fr) La «biture express » également baptisée binge drinking dans les pays anglosaxons $<$ sic $>[\ldots]$

(57fr) L'intoxication alcoolique aiguë, phénomène connu sous le nom de «binge drinking » [...]

(58fr) La biture express ou «binge drinking » $[\ldots]$

Dans le cas de la langue polonaise c'est binge drinking qui est toujours l'élément qui nécessite une explication et non pas l'inverse. Le corpus tchèque ne contient aucune attestation de ce phénomène, le terme anglais est toujours expliqué par la suite, comme montré dans les exemples dans les catégories 1 et 2.

\subsection{Indication de l'origine}

Dans le même ordre d'idée d'une formulation maladroite, un raccourci d'expression laisse entendre, par le recours à vient de utilisé couramment en étymologie, que binge drinking serait l'étymon de biture express (59fr), ce qui est d'évidence une contre-vérité, même s'il est tout aussi évident que la création en français du néologisme biture express s'est faite pour compenser le manque dans cette langue d'une dénomination correspondant au phénomène apparu dans les pays anglo-saxons et dénommé binge drinking - et parfois mais beaucoup plus rarement speed drinking -, terme qui était également récent dans les pays anglophones.

(59fr) Biture express vient de l'anglais « binge drinking ».

Dans les articles en polonais, le terme binge drinking est lié à une mode venue des pays anglo-saxons, les articles dans lesquels cette locution apparaît avertissent du danger de cette " mode ", ce qui indique que ce terme est plutôt récent et relativement peu connu.

Dans le corpus tchèque, l'origine de l'expression est souvent mentionnée dans les articles expliquant le phénomène de binge drinking, mais c'est plutôt au niveau des acteurs (les Britanniques, les Anglo-Saxons, la littérature américaine, etc.), que la langue anglaise est moins souvent mentionnée explicitement : 
(18tch) [...] problém nezřízeného víkendového opíjení, pro které má angličtina výraz binge drinking... [...le problème de la consommation sans contrôle pendant les week-ends, pour laquelle l'anglais possède l'expression binge drinking.... $]^{50}$.

\subsection{Traduction par une tierce langue}

Le corpus tchèque permet de dévoiler une catégorie à part, où l'équivalent de l'anglicisme binge drinking est expliqué pour les lecteurs tchèques en se servant d'une autre langue, en l'occurrence de l'allemand.

(19tch) Má i speciální název - v anglicky mluvícím prostředí se hovoří o binge drinking, němčina užívá výraz Rauschtrinken. Česky bychom ho mohli označit jako pití při flámu. [Il possède même son propre terme - dans le milieu anglophone, on parle de binge drinking, l'allemand utilise l'expression Rauschtrinken. En tchèque, on pourrait le décrire comme la consommation lors d'une fête $]^{51}$.

Ce choix n'est pas anodin puisque le mot rauš est un vieux emprunt à l'allemand (Rausch) qui s'utilise notamment dans le discours informel/argotique dans la locution být v rauši (= "être dans un délire ») et illustre alors le caractère plutôt familier du composé anglais.

\subsection{Cas complexes}

Enfin, on peut noter des formulations complexes combinant plusieurs des structures ou outils mentionnés dans les sections précédentes. Ainsi, pour ne prendre qu'un exemple, (60fr) cumule le recours à un verbe de dénomination, appelé, et deux dénominations jointes par la conjonction de coordination ou, mais celle-ci qui met en relation l'emprunt et l'équivalent, les place dans l'ordre inverse de l'ordre logique et chronologique attendu, faisant apparaître l'équivalent néologique français avant le terme anglais qui l'a suscité, comme dans les exemples des sections 7. à 9.

(60fr) On observe cette manière d'alcoolisation, appelée « biture express » ou « binge drinking [...]

\section{Un mot sur la lexicalisation}

Les configurations 7, 8 et 9 ont été présentées comme indicatrices d'un certain degré de lexicalisation, puisqu'elles présentent l'équivalent autochtone comme le terme " par défaut ». L'analyse de nos trois corpus montre que ce type de lexicalisation est limité en français et quasi-absent en polonais et en tchèque. L'absence dans ces deux dernières langues ne s'explique sans doute pas par une pénétration moindre de ce phénomène

\footnotetext{
Reflex, 2004 (hebdomadaire).

${ }^{51}$ Psychologie dnes, 2014 (mensuel).
} 
dans la société polonaise ou tchèque, mais il est plutôt question d'un attachement aux équivalents locaux qui ne laissent pas de lacune pour la pénétration de binge drinking comme enrichissement du champ lexical de la «cuite». Pour le français, toutefois, la lexicalisation n'est pas négligeable. Les archives du Monde font état de 26 articles où figure biture express, l'équivalent le plus fréquent de binge drinking, y compris dans quelques (rares !) articles où l'anglicisme est absent : «Sunny Beach, ou le paradis (artificiel) des jeunes Européens adeptes de la biture express et des discothèques tapageuses ${ }^{52}$ ». Il entre également en composition ( Génération 'biture express' » ${ }^{53}$ ).

Dans les langues polonaise et tchèque, il est peu probable que binge drinking s'impose face aux équivalents locaux et pratiques locales. Les textes de presse dans lesquels les occurrences de ce phénomène ont été repérées sont surtout des articles parlant de modes juvéniles ou des dangers liés aux mœurs contemporaines. Binge drinking par ailleurs apparaît surtout dans les revues consacrées à la santé (psychologie) et dans les articles scientifiques (qui ne sont pas inclus dans le cas de cette recherche) du domaine de la sociologie et de la médecine. Il est peu probable que cette expression se maintienne, à l'opposé de binge watching, qui a trouvé un créneau faute d'équivalent local. Après tout, la pratique est connue depuis la nuit des temps : il suffit de mentionner l'expression de l'époque napoléonienne "Saoul comme un Polonais» ou le classement des pays en fonction de la consommation de bière par habitant ${ }^{54}$ pour voir quelles nations détiennent la palme des experts dans le domaine de la cuite. Emprunter cette expression aux Anglais est l'équivalent d'apprendre le jeu de dames à des grands maîtres d'échec.

\section{Conclusion}

La mise en relation d'un emprunt et d'un ou de plusieurs équivalents autochtones est un phénomène que l'on observe fréquemment. Et celle-ci va dans les deux sens, mais le plus fréquent, au moins au début, est d'avoir d'abord l'emprunt suivi d'un ou plusieurs équivalents (cas de foisonnement néologique, voir infra). Le sens peut s'inverser quand un équivalent s'est largement implanté dans la langue. Autrement dit, le segment glosateur apporte des informations sur le degré de lexicalisation non seulement de l'emprunt, mais aussi de l'équivalent, surtout lorsque celui-ci est manifestement néologique. L'emprunt peut ne venir alors qu'en deuxième position uniquement comme rappel de l'origine de la réalité en question avec sa dénomination étrangère.

Plusieurs preuves attestent de l'intégration dans la langue et d'une vaste circulation de binge drinking, particulièrement en français. On relève des ellipses de drinking dans des soirées binge, des «binge party»... Par ailleurs, on constate le développement d'une famille morphologique formée sur cet emprunt : nom d'acteur avec binge drinker, verbes dans ils binge-drinkent, et avec une ellipse : se binger. Dans les langues polonaise et tchèque, ce sont les équivalents locaux qui prennent le dessus. Toutefois, on atteste l'existence

5231 mai 2018 https://www.lemonde.fr/economie/article/2018/05/30/a-sofia-le-tourisme-a-tout -prix_5306658_3234.html

5303 juin 2013 https://www.lemonde.fr/culture/article/2013/06/03/le-formidable-succes-de-stromae 3423010_3246.html.

54 https://fr.wikipedia.org/wiki/Consommation_annuelle_de_bi\%C3\%A8re_par_habitant_par_pays. 
du substantif pour désigner : binge-drinker (en tchèque, avec même le pluriel adapté : binge-drinkeři) celui qui s'adonne à cette conduite. Les déclinaisons de binge drinking(- $u$, -em) témoignent du processus d'adaptation lente de l'emprunt vers le tchèque ; de manière semblable également vers le polonais.

Enfin, cet emprunt sert de modèle à d'autres créations comme speed drinking, binge watching, binge eating, binge shopping, etc.

Toutefois, on constate des indices de lexicalisation de deux ordres : soit des remarques métalinguistiques, soit des équivalents présentés comme la dénomination non marquée. On relève des commentaires comme «traduit en français bien de chez nous »; " plus communément appelé chez nous " cuite express »; la " beuverie express » est désormais l'expression française ; [Binge drinking] a désormais son équivalent en bon français, « beuverie express »..., formules que l'on relève moins en polonais ou en tchèque, sans qu'elles soient complètement absentes. L'emprunt s'implante en français, mais les locuteurs valorisent aussi les formes autochtones. On note ici un moindre foisonnement néologique, les formes préférées incorporant l'élément express. Quant à l'ordre de présentation, les exemples (48fr) à (50fr) («cuite express (binge drinking) » et encore plus (52fr) à (54fr) "biture express ", encore appelée "binge drinking ") témoignent de la prégnance de l'équivalent français. Le polonais et le tchèque accusent bien moins d'exemples des équivalents faisant partie des catégories plus assimilées, ce qui reflète non de la moindre pénétration de ce type de comportement dans les sociétés concernées mais de la moindre médiatisation du risque de ces comportements par les journalistes des deux pays.

La ponctuation et, plus généralement, les astuces typographiques fournissent également des marqueurs d'assimilation, à la fois pour l'emprunt et l'équivalent - moins il y a de signes comme les guillemets ou l'italique, plus la lexie est assimilée - mais le corpus aléatoire exploité ne permet pas une étude statistique.

Dans la mise en relation entre un emprunt et un ou des équivalents autochtones, il existe toute une palette de moyens linguistiques - des plus explicites à de simples constructions détachées indiquées par des signes typographiques - à la disposition des locuteurs qui choisissent tel ou tel moyen, et en combinent parfois plusieurs. L'examen de ces relations, exprimées dans les gloses, permet d'apprécier les stratégies d'appropriation à la fois de l'emprunt et de l'équivalent, indice de la lexicalisation des deux identités. Si l'emprunt prend manifestement racine dans les trois langues, les équivalents, qui sont nombreux surtout en français, prennent une certaine place dans le lexique.

Dans les corpus polonais et tchèque, les constructions les plus fréquemment attestées sont celles où binge drinking se retrouve expliqué par un équivalent à l'aide de l'introduction de c'est-à-dire ou donc (configuration 4). Pour ce qui est des constructions détachées, il n'y a guère d'appositions du type « équivalent entre parenthèses » (configuration 8) ou, uniquement pour le polonais, $d^{\prime}$ " emprunt en apposition après équivalent » (configuration 5). Cependant, on retrouve un grand nombre de constructions détachées du type « emprunt entre parenthèses après équivalent " (configuration 7), indice d'un degré d'intégration moindre en polonais et en tchèque par rapport au français. 


\section{BIBLIOGRAPHIE}

Depecker, L. (2001) : L'invention de la langue, Le choix des mots nouveaux. Paris : Larousse et Armand Colin. Hildenbrand, Z., Kacprzak, A. et Sablayrolles, J.-F. (éds) (2016) : Emprunts néologiques et équivalents autochtones en français, en polonais et en tchèque, Limoges : Lambert-Lucas.

Pflanz, M.-L. (2014) : « Emprunt lexical : existe-t-il une typologie de la phase néologique ? ", Neologica 8, pp. 157-183.

Rey-Debove, J. (1998) : La linguistique du signe. Une approche sémiotique du langage. Paris : Armand Colin.

Sablayrolles, J.-F. et Napieralski, A. (2016) : «Emprunts sociétaux : conduites « délictueuses, excessives ou à risque » en français et en polonais ». In Hildenbrand, Z., Kacprzak, A. et Sablayrolles, J.-F. (2016) : Emprunts néologiques et équivalents autochtones en français, en polonais et en tchèque. Limoges : Lambert-Lucas, Collection La lexicothèque, pp. 89-156.

Steuckardt, A. et Honoré, J.-P. (2006) : L'emprunt et sa glose. Aix-en-Provence : Presses de l'Université de Provence.

Steuckardt, A. et Niklas-Salminen, A. (2003) : Le mot et sa glose. Aix-en-Provence : Presses de l'Université de Provence.

Steuckardt, A. et Niklas-Saliminen, A. (éds) (2005) : Les marqueurs de glose. Aix-en-Provence : Publications de l'Université de Provence.

\section{Sitographie}

Kř̌en, M., Cvrček, V., Čapka, T., Čermáková, A., Hnátková, M., Chlumská, L., Jelínek, T., Kováŕíková, D., Petkevič, V., Procházka, P., Skoumalová, H., Škrabal, M., Truneček, P., Vondřička, P. et Zasina, A. (2019) : Korpus SYN, verze 8 z 12. 12. 2019, Ústav Českého národního korpusu FF UK, Praha, https://www.korpus.cz. https://www.sketchengine.eu/

http://aranea.juls.savba.sk/

Jean-François Sablayrolles

UMR 7597 Histoire des théories linguistiques

Alena Podhorná-Polická

Ústav románských jazyků a literatur Filozofické fakulty

Masarykova univerzita

Arna Nováka 1, Brno 60200

podhorna@phil.muni.cz

Andrzej Napieralski

Wydział Filologiczny

Université de Łódź

Ul. Pomorska 171/173, 90-236, Łódź

andrzej.napieralski@uni.lodz.pl

John Humbley

CLILLAC-ARP EA 3967

Université de Paris,

Case 7002, 75205 Paris cedex 13

humbley@eila.univ-paris-diderot.fr 